\title{
Imaginary optical constants in near-infrared (NIR) spectral range for the separation and discrimination of adulterated diesel oil binary mixtures
}

\author{
Boniphace Kanyathare ${ }^{1}$ Benjamin Asamoah ${ }^{1} \cdot$ Kai-Erik Peiponen $^{1}$
}

Received: 10 July 2018 / Accepted: 29 November 2018 / Published online: 11 December 2018

(c) The Author(s) 2018

\begin{abstract}
Fuel adulteration is a major factor contributing to environmental pollution. The tendency to adulterate diesel oil with kerosene is becoming prevalent in many countries, which is particularly driven by the lower cost of kerosene than of diesel oil. The objective of this study is to develop a method of separating and discriminating between different compositions of kerosene in binary liquid fuel mixtures. In this study, we have utilized refractive indices of binary fuel mixtures measured using an Abbe refractometer, and the inversion of measured transmittance spectra in the visible and near-infrared (NIR) spectral range by a general method based on Kramers-Kronig dispersion analysis, where the singly subtractive Kramers-Kronig relations have been used to obtain the wavelength-dependent imaginary excess permittivity of a binary liquid mixture. The excess permittivity has considerable importance in the interpretation of different liquid molecule interactions in binary liquid mixtures. It is herein demonstrated that the problematic cases of relatively low adulterations of 5\% and 10\% can be detected and distinguished from $15 \%$ adulteration using imaginary optical properties in the NIR spectral range. We show that imaginary optical constants such as excess imaginary permittivity and the extinction coefficient are useful for not only screening for adulteration, but also discriminating and separating low from high kerosene compositions in mixtures. Moreover, these quantities can also be exploited for the development of practical sensors, especially for sensing diesel oil adulteration under field conditions.
\end{abstract}

Keywords Diesel oil $\cdot$ Liquid fuel adulteration $\cdot$ Near-infrared spectroscopy $\cdot$ Refractive index $\cdot$ Singly subtractive Kramers-Kronig relation $\cdot$ Imaginary excess permittivity

\section{Introduction}

Liquid fuel adulteration is becoming a common problem in many countries. This unethical practice is carried out to increase profit. The underlying reason for this practice is the uneven taxation system for different liquid fuels, where kerosene is generally subsidized, while diesel and gasoline have the higher tax rates. This illegal practice leads to adverse effects such as damage to engines, loss of taxation

Boniphace Kanyathare

elphace.boniphace@gmail.com

$\triangle$ Kai-Erik Peiponen

kai.peiponen@uef.fi

1 Department of Physics and Mathematics, University of Eastern Finland, P.O. Box 111, 80101 Joensuu, Finland for authorities, increased traffic accidents, and environmental pollution resulting from exhaust gas emissions $[1,2]$.

The most common form of fuel adulteration in many parts of the world is to add kerosene to either diesel oils or gasoline. These fuels consist of hydrocarbons, which have spectral fingerprints in the near-infrared (NIR) spectral region. Nevertheless, it is challenging to screen diesel oils for adulteration with kerosene, especially when the content of the adulterants tends to be below $20 \%$, using NIR spectra owing to the closely overlapping fingerprints of the two liquids. However, the development of optical measurement methods for adulterated fuels is feasible because of the different optical properties of these liquid fuels, which can usually be detected rapidly by different and relatively simple measurement devices. Furthermore, it is possible to perform these measurements in the field. Both spectral and non-spectral optical measurement methods have been proposed as methods for screening adulterated fuel [3-6]. 
For the rapid optical evaluation of fuel quality, an Abbe refractometer is a useful device for screening adulterated diesel oils via the refractive index [6]. However, there are cases, where the refractive index of diesel oil is very close to that of kerosene, and such a case is demonstrated in this study. Therefore, under such circumstances further data, in addition to the refractometer reading, is required to confirm sample adulteration. In our previous work, we have demonstrated the use of a prototype hand-held sensor for the detection of diesel oils adulterated with kerosene. The prototype is based on a modified commercial hand-held glossmeter. However, the measurement time with this type of sensor is still long compared with the almost instant reading with an Abbe refractometer or the relatively rapid NIR measurement with a (portable) spectrophotometer. Another type of hand-held sensor for screening adulterated diesel oils is based on an Abbe refractometer and a data analysis method using the real part of excess permittivity with a fixed probe wavelength [7]. Regarding field measurements, NIR spectrum measurement has also been tested for crude oil quality inspection under difficult field measurement conditions [8].

In our recent studies of adulterated diesel oils, we have learned that when using either an Abbe refractometer or a spectrophotometer, there are cases when one may need additional complementary methods to reliably confirm diesel oil adulteration. To circumvent this issue, we have proposed a combination of both refractive index and NIR transmittance measurements, along with exploitation of the so-called excess permittivity of a binary mixture to obtain a more comprehensive picture of adulterated diesel oils [9]. In other words, we have shown that the real part of the complex excess permittivity of adulterated diesel oil reveals fine structures of spectral fingerprints that can be used to screen adulterated diesel oil. The study reported in this paper is a continuation of [9], namely, we have investigated the imaginary part of the complex excess permittivity of authentic and adulterated diesel oils. It is shown that the combination of the real part of the refractive index, the imaginary part of the refractive index (obtained from the absorption coefficient of the fuel), and the imaginary part of the excess permittivity of adulterated diesel oils, provides a plausible means of deciding with confidence the relative amounts of kerosene adulteration in diesel oils.

To exploit the imaginary excess permittivity in adulterated diesel oil, we utilize the complex-valued excess relative permittivity, which is a useful measure for studying the difference between the measured and ideal permittivities of a liquid mixture. This relative excess permittivity is related to the complex refractive index of a binary liquid. Furthermore, the excess permittivity can be used as a measure of the interaction between molecules of different liquid components in binary liquid mixtures $[10,11]$. As examples of this type of application, it is possible to assess the validity of different mixing rules for the optical constants of binary liquids by studying perturbed hydrogen bonding in the IR region and the corresponding excess absorbance [12], and to study the excess absorbance of water-alcohol mixtures as well as ionic liquid mixtures in the terahertz (THz) gap [13, 14]. The theoretical foundations of the complex excess permittivity which depends on the frequency of the incident electromagnetic field (the frequency-dependent complex excess permittivity is crucial in our case), have been derived relatively recently for binary and multiple liquid mixtures [15]. In this paper, we are concerned with the imaginary part of the wavelength-dependent excess permittivity. The complex permittivity of a binary liquid mixture depends on the displacement of electrons (light interaction) and molecules (IR interaction), and the molecular structure, size, and weight of the molecules. These factors are beyond the scope of these studies, because there are hundreds of different hydrocarbons in diesel oils.

Here, we report, to the best of our knowledge, the first ever study on liquid fuel adulteration based on imaginary excess permittivity focusing on diesel oil adulteration with different volume fractions of kerosene. The imaginary excess permittivity is calculated using NIR transmission spectra measured with a spectrophotometer, the refractive index measured with an Abbe refractometer, and using the singly subtractive Kramers-Kronig (SSKK) relation [16] to obtain an estimate of the wavelength-dependent refractive index, which is needed in the calculation of the imaginary excess permittivity. The basis of the SSKK relation is a rather fundamental property of physics and chemistry, namely, causality [16]. Therefore, SSKK analysis has general validity and can be used regardless of the spectral line shape model assumed for. The validity of the method is exemplified using diesel of two different grades adulterated with kerosene of the same grade.

To provide a more comprehensive picture of screening problematic adulterated diesel oils, here, we study the extinction coefficient and imaginary excess permittivity. The former is obtained directly by measurement, whereas the latter is calculated.

\section{Theory}

The imaginary excess permittivity can be derived from the equation for the excess permittivity of binary mixtures. If we denote the complex relative permittivity of diesel oil by $\varepsilon_{\mathrm{D}}$ and the corresponding permittivity of kerosene by $\varepsilon_{\mathrm{K}}$, we can express the complex excess relative permittivity (here after we use the expression for permittivity, meaning that it is the dimensionless relative permittivity) as [9]

$$
\varepsilon^{\mathrm{E}}=\varepsilon-\varepsilon^{\text {ideal }}=\varepsilon-\varepsilon_{\mathrm{K}}\left[1+f_{\mathrm{D}}\left(\left(\frac{\varepsilon_{\mathrm{D}}}{\varepsilon_{\mathrm{K}}}\right)-1\right)\right] .
$$


Here, $\varepsilon$ is the complex permittivity obtained from optical measurements, $\varepsilon^{\text {ideal }}$ is the complex ideal permittivity of the binary mixture without any interactions between unlike liquid molecules, $f_{\mathrm{D}}$ is the volume fraction of diesel oil, and $\varepsilon_{\mathrm{D}} / \varepsilon_{\mathrm{K}}$ is the permittivity ratio. The sign and magnitude of $\varepsilon^{\mathrm{E}}$ are a measure of the strength of interactions between molecules in a binary mixture. There are hundreds of different hydrocarbons in diesel oil [17], and after adulteration by mixing with different percentages of kerosene, the dipole moments of the electric charges of the binary mixture will change, causing small but observable differences in the measured spectral features. This change also leads to differences in the real and imaginary parts of the excess permittivity, which is the key idea of this paper. The ideal permittivity in Eq. (1) corresponds to the upper Wiener bound [18] of an effective medium as follows:

$\varepsilon^{\text {ideal }}=f_{\mathrm{D}} \varepsilon_{\mathrm{D}}+\left(1-f_{\mathrm{D}}\right) \varepsilon_{\mathrm{K}}$.

In general, the expression for the frequency-dependent complex permittivity of a homogeneous medium is given by

$\varepsilon(\omega)=\varepsilon_{1}(\omega)+i \varepsilon_{2}(\omega)=n^{2}(\omega)-k^{2}(\omega)+i 2 k(\omega) n(\omega)$,

where $\omega$ is the angular frequency of incident electromagnetic radiation and $i$ is the imaginary unit. The complex refractive index is given by $N(\omega)=n(\omega)+i k(\omega)$, where $n$ is the conventional refractive index and $k$ is the extinction coefficient of the medium. The complex relative permittivity of an insulator such as a pure liquid or one of the binary mixtures in this study is given by $\varepsilon=N^{2}$, which was used in the second equality in Eq. (3).

Using the first equality in Eq. (1) and second equality in Eq. (3), the imaginary excess permittivity is given by

$$
\begin{aligned}
\operatorname{Im} \varepsilon^{E}(\lambda) & =2 n(\lambda) k(\lambda)-2 n_{\text {ideal }}(\lambda) k_{\text {ideal }}(\lambda) \\
& =2 n(\lambda) k(\lambda)-2\left(f_{\mathrm{D}} n_{\mathrm{D}}(\lambda) k_{\mathrm{D}}(\lambda)+\left(1-f_{\mathrm{D}}\right) n_{k}(\lambda) k_{k}(\lambda)\right),
\end{aligned}
$$

where $\lambda$ is the wavelength of light, $n(\lambda)$ and $k(\lambda)$ are the wavelength-dependent refractive index and extinction coefficient of an adulterated sample, respectively. In our case, $n_{\text {ideal }}(\lambda)$ and $k_{\text {ideal }}(\lambda)$ are the wavelength-dependent refractive index and extinction coefficients of ideal binary mixtures, $n_{\mathrm{D}}(\lambda)$ and $k_{\mathrm{D}}(\lambda)$ are the wavelength-dependent refractive index and extinction coefficient of authentic diesel oil, and $n_{\mathrm{K}}(\lambda)$ and $k_{\mathrm{K}}(\lambda)$ are the wavelength-dependent refractive index and extinction coefficient of kerosene, respectively. The wavelength-dependent refractive indices and extinction coefficients of authentic diesel oil and kerosene, $n_{\mathrm{D}}(\lambda)$, $k_{\mathrm{D}}(\lambda)$, and $n_{\mathrm{K}}(\lambda), k_{\mathrm{K}}(\lambda)$, as well as those of adulterated diesel oils $n_{\mathrm{K}}(\lambda)$ and $k_{\mathrm{K}}(\lambda)$, respectively, were obtained in several steps. First, the transmission spectra and refractive index of the sample were measured, then, Lambert's intensity law
(Eq. 5), the extinction coefficient (Eq. 6), and the SSKK relation (Eq. 7) were applied.

Lambert's intensity law is given as

$I(\lambda)=I_{0}(\lambda) \mathrm{e}^{-\alpha(\lambda) d}$,

from which one obtains the wavelength-dependent absorption coefficient $\alpha$ of the medium for an a priori known sample thickness $d$. Hence, we can calculate $k$ from the formula:

$k(\lambda)=\frac{\lambda \alpha(\lambda)}{4 \pi}$.

In this work, we exploited the wavelength of Vis-NIR radiation rather than the angular frequency. Because we can measure the discrete refractive index for an anchor point with an Abbe refractometer, we further utilize the SSKK dispersion relation to obtain $n$ using [16]

$n\left(\lambda^{\prime}\right)-n\left(\lambda^{\prime \prime}\right)=\frac{2\left(\lambda^{\prime 2}-\lambda^{\prime \prime 2}\right)}{\pi} P \int_{0}^{\infty} \frac{\lambda k(\lambda) \mathrm{d} \lambda}{\left(\lambda^{2}-\lambda^{\prime 2}\right)\left(\lambda^{2}-\lambda^{\prime \prime 2}\right)}$.

Here, $n\left(\lambda^{\prime}\right)$ is the wavelength-dependent refractive index, $n\left(\lambda^{\prime \prime}\right)$ is a priori known refractive index at an anchor point $\lambda^{\prime \prime}$, and $P$ denotes the Cauchy principal value. To the best of our knowledge, this is the first time that Kramers-Kronig analysis has been applied to the wavelengthdependent imaginary excess permittivity of binary liquid mixtures. In this work, the fixed wavelength $\lambda^{\prime \prime}$ is the same as the wavelength of the Abbe refractometer, namely, $589 \mathrm{~nm}$. In the practical calculation, the integral, especially in the case of SSKK analysis, can usually be truncated to correspond to the initial and final points of the measured transmittance. Here, we use a method to optimize the anchor point $\lambda^{\prime \prime}$ to minimize the possible data inversion error. This method is based on the properties of a Chebyshev polynomial shown by Palmer et al. [19]. Because of the fixed wavelength of the Abbe refractometer, we are not free to choose the anchor point. Nevertheless, we employ the optimal anchor point by fixing the wavelength of the final point $\left(\lambda_{\mathrm{f}}\right)$ of the transmittance spectrum and we calculate the initial wavelength $\left(\lambda_{\mathrm{i}}\right)$ that corresponds to the optimal anchor point. The wavelength $\lambda_{\mathrm{i}}$ is then obtained from a zero of a Chebyshev polynomial of the first kind as follows [19]:

$\lambda_{\mathrm{i}}=\frac{\lambda_{\mathrm{f}} \lambda^{\prime \prime}}{\sqrt{2 \lambda_{\mathrm{f}}^{2}-\lambda^{\prime \prime 2}}}$.

Hence, the scanning of the transmittance spectrum was started from the calculated wavelength. The SSKK method has been used, for example, to analyze the properties of diesel oil droplets [20], but not to investigate the imaginary part of the excess permittivity of adulterated diesel oils. In the 
SSKK method, the location of the anchor point is not usually a critical factor for the successful inversion of extinction coefficient data.

\section{Materials and methods}

In this work, we studied summer-grade (sample A) and winter-grade [sample $\mathrm{B}\left(-20^{\circ} \mathrm{C}\right)$ ] diesel oils that originate from the same crude oil, to take into account different climatic conditions throughout the year in different countries. The first very important additive in diesel oils (both summergrade and winter-grade) is wax, which is normally added to increase the combustion speed leading to more power owing to its higher cetane value. However, during winter (low operating temperatures), wax tends to solidify and cause problems during ignition; therefore, anti-wax additives are added to avoid solidification and enable smooth engine operation. Thus, the main difference between the summer and winter diesel compositions is the inclusion of anti-wax additives, which are present in winter-grade diesel oils but not in summer-grade diesel oils. The value of $-20^{\circ} \mathrm{C}$ is the lowest temperature at which a diesel engine is expected to start. The kerosene sample in this study is a low-odor commercial product (sample C, Alfa Aesar). It is important to emphasize the variation of the optical properties of diesel oil and kerosene from different oil fields, because these are complex liquids with hundreds of hydrocarbons [17]. Owing to this fact, we chose a well-known laboratory quality kerosene as a reference that, in principle, is available to scientists worldwide. Obviously, the refractive index of the diesel oil and kerosene depends on the origin of the crude oil from which the fuel samples were refined.

The refractive index of pure and adulterated diesel oil was measured at room temperature of $22.7 \pm 0.02{ }^{\circ} \mathrm{C}$ using an Abbe refractometer (Atago RX5000) having an operating wavelength of $589 \mathrm{~nm}$ and a relatively high accuracy of \pm 0.00004 refractive index units. Differences in the measurement temperature will affect the refractive index reading owing to changes in the thermo-optic coefficient of the fuel sample. Therefore, it is important in the field measurements to pay attention to the temperature variations to avoid possible misinterpretation of results.

The NIR transmittance spectra of the samples were measured at a room temperature of $23{ }^{\circ} \mathrm{C}$ using spectrophotometer (PerkinElmer Lambda 9) and a 10-mm-thick quartz cuvette. The thick cuvette was used to test the applicability to various field conditions.

Before recording the initial spectra of the fuel samples, a baseline correction measurement was performed by recording the spectra of an empty cuvette: this baseline was recorded in the memory of the software of the spectrophotometer and was automatically considered in subsequent measurements. Furthermore, multiplicative scatter correction (MSC) pretreatment was performed on the spectral data using PLS-Toolbox (Eigenvector Research, Inc USA) in the MATLAB software, to minimize or eliminate Pathlength-related errors, baseline shifts, and interference. To ensure that our spectral data were suitable for further processing, we compared the pretreatment in MATLAB with MSC pretreatment carried out using UnscramblerX (CAMO, Norway). The spectrophotometer measures transmittance in the UV-Vis-NIR range with an accuracy of $0.07 \%$ for an absorbance value equal to 1 . However, the target spectral range for this work was the Vis-NIR range. Our choice of wavelengths of considerably below $2000 \mathrm{~nm}$ for the spectra was based on the availability of such portable spectrometers on the market suitable for the detection of hydrocarbons in diesel oils which operate below $2000 \mathrm{~nm}$. We remark that, during these studies, we also measured the transmittance using 10-mm-thick and 5-mm-thick cuvettes filled with fuel samples and observed the transmittance ratio from the obtained transmittance spectra to avoid any discrepancy of data due to an empty cuvette. However, we did not find any significant discrepancy for the samples of this study. For our target field measurement conditions, the utilization of a single cuvette, especially one with a thickness of $10 \mathrm{~mm}$, is more practical than that of two cuvettes, because the former cuvettes are cheaper and can be easily rinsed than $5 \mathrm{~mm}$ and $1 \mathrm{~mm}$ cuvettes. Nevertheless, because of possibility of contamination of the cuvette during the field measurements, specially designed single cuvette with two different optical path lengths that incorporates a beam splitter to guide the probe beam via the two paths, and thus provides the transmittance ratio would be useful. Such a procedure would help to eliminate noise due to possible contamination of the cuvette.

\section{Results and discussion}

We show the refractive index data for pure fuel samples A, $\mathrm{B}$, and $\mathrm{C}$, measured by the Abbe refractometer at $589 \mathrm{~nm}$ in Table 1. It is clear from Table 1 that the summer-grade diesel oil (sample A) has a higher refractive index than the winter-grade diesel oil (sample B) and the kerosene (sample C). Furthermore, the refractive indices of samples B and C differ only in the third decimal place. The refractive index of kerosene sample $\mathrm{C}$ is very close to that of winter-grade diesel oil sample B, which indicates the problem that one can expect only a minute difference in the refractive index, between the authentic and adulterated diesel oils.

Table 1 also gives the refractive indices of the adulterated diesel oils. In all cases, the refractive index of the binary mixture decreases with increasing kerosene volume fraction. For the binary mixtures for sample A, the refractive index 
Table 1 Measured refractive index data for authentic diesel oil, kerosene, and their mixtures at $589 \mathrm{~nm}$

\begin{tabular}{lll}
\hline Sample & $\begin{array}{l}\text { Volume percentage of kero- } \\
\text { sene }\end{array}$ & $\begin{array}{l}\text { Adulterated sample } \\
n\end{array}$ \\
\hline Summer-grade diesel oil (sample A) & $0 \%$ & 1.46353 \\
& $5 \%$ & 1.46195 \\
& $10 \%$ & 1.46084 \\
Winter-grade diesel oil (sample B) & $15 \%$ & 1.45941 \\
& $0 \%$ & 1.44775 \\
& $5 \%$ & 1.44884 \\
Kerosene (sample C) & $10 \%$ & 1.44793 \\
\hline
\end{tabular}

readings for $5 \%, 10 \%$, and $15 \%$ kerosene become increasingly close to that of diesel oil sample A. These readings are always between those of sample A and kerosene, in line with the conventional binary mixture model. However, the mixing rules, such as those in [12], for an ideal binary liquid are not valid for these samples. Indeed, we have shown, for example, that the Lorentz-Lorenz (L-L) formula is invalid for the present samples. Thus, the permittivity ratio, especially with increasing amount of kerosene, does not linearly depend on the kerosene fill fraction. Hence, the quantification of the kerosene fill fraction using the $\mathrm{L}-\mathrm{L}$ formula is problematic.

For sample B adulterated with 5\% and 10\% kerosene, the refractive indices are slightly above the reading of pure diesel oil, whereas that for sample B adulterated with $15 \%$ kerosene is slightly below it. In fact, this behavior for sample B is contrary to that usually expected for binary mixtures, namely, under ideal conditions, the refractive indices of mixtures are expected to lie between those of the two mixture constituents. Sample B adulterated with $15 \%$ kerosene could be erroneously concluded to be authentic diesel oil if and only if the refractive index reading is considered, namely, $1.44731(15 \%)$ and $1.44775(0 \%)$ are close to each other. When the amount of authentic diesel oil in the $15 \%$ adulterated sample is increased to reduce the adulteration to $10 \%$ and finally $5 \%$, the abnormal behavior of the refractive index becomes evident, and hence, such a dilution process for the originally $15 \%$-adulterated diesel oil increases the confidence in the judgement that the diesel has been adulterated. In our recent article [7], we demonstrated that sometimes when the refractive indices values for both authentic and adulterated samples are very similar, it might lead to authentic diesel erroneously being interprated as adulterated. Therefore, a more rigorous measure is required to ascertain the differences between samples. Moreover, in the same article, the insignificant effect of small temperature variations on the refractive index was explained. The article placed more emphasis on the role of chemical interactions in invalidating the $\mathrm{L}-\mathrm{L}$ formula if one attempts to quantify the amount of kerosene. These interactions are the cause of nonlinear variations of the refractive index, especially for the present case of diesel oils adulterated with kerosene [7].

Figure 1 shows the transmittance curves of pure fuel sample A together with the corresponding adulterated samples in the spectral range of 431-1600 nm. The wavelength of $1600 \mathrm{~nm}$ was fixed, and that of $431 \mathrm{~nm}$ was calculated from Eq. (8). Diesel oil and kerosene also have a strong absorption band in the range of approximately 1700-2100 nm [21], but we have omitted this band as well as bands in the midinfrared spectral range. As shown in Fig. 1, the curves for authentic diesel oil (sample A) and kerosene (sample C) overlap. However, it is possible to clearly differentiate the authentic diesel oil from the adulterated samples, whereas it is difficult to differentiate the adulterated samples. For the sample with $5 \%$ adulteration, it is possible to differentiate the sample from other adulterated samples, especially at visible wavelengths. Moreover, the corresponding transmittance curves for $10 \%$ and $15 \%$ adulteration show even greater overlap in Fig. 1. For sample B, the screening of adulterated diesel oils is much more difficult regarding the

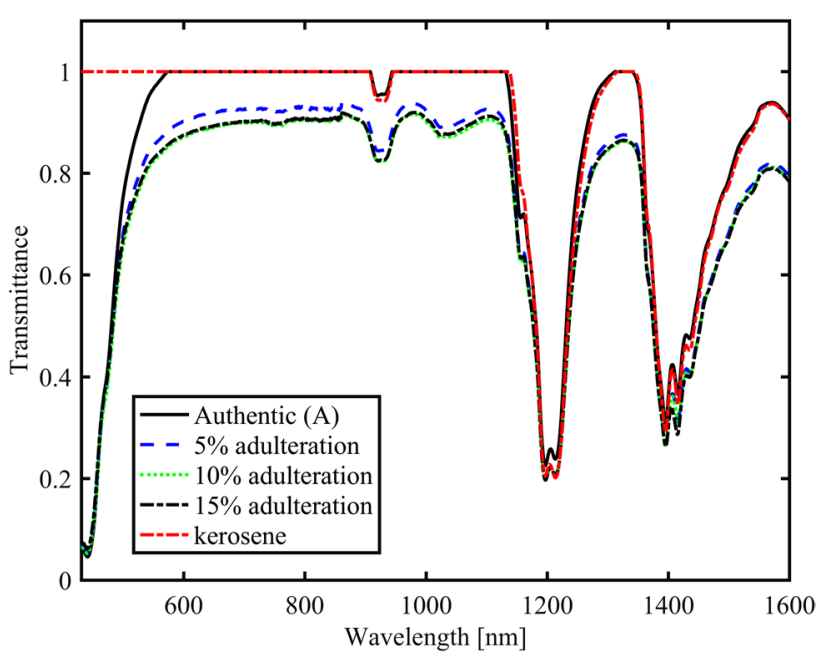

Fig. 1 Transmittance curves for authentic and adulterated samples A and $\mathrm{C}$ 
interpretation of the spectral data, and its refractive index is close to that of sample $\mathrm{C}$. This again shows the difficulty of screening adulterated diesel oils using their Vis-NIR spectra and refractive index.

From the measured transmittance spectra of the pure and adulterated samples, we calculated the wavelength-dependent extinction coefficient of the samples using Eq. (6). Figure $2 \mathrm{a}, \mathrm{b}$ shows magnifications of extinction coefficient curves for kerosene, and authentic and adulterated samples $\mathrm{A}$ and $\mathrm{B}$ in the vicinity of the strong absorption band ranging from 1190 to $1220 \mathrm{~nm}$. It is evident from Fig. 2a, b that the shoulder of the fingerprint of the pure diesel oils at $1195 \mathrm{~nm}$ is higher than that at $1214 \mathrm{~nm}$, whereas the opposite holds for the kerosene. Moreover, pure kerosene and diesel oil can already be identified from the relative heights of the two spectral peaks of these samples. However, when one mixes kerosene with diesel oils $\mathrm{A}$ and $\mathrm{B}$, the presence of
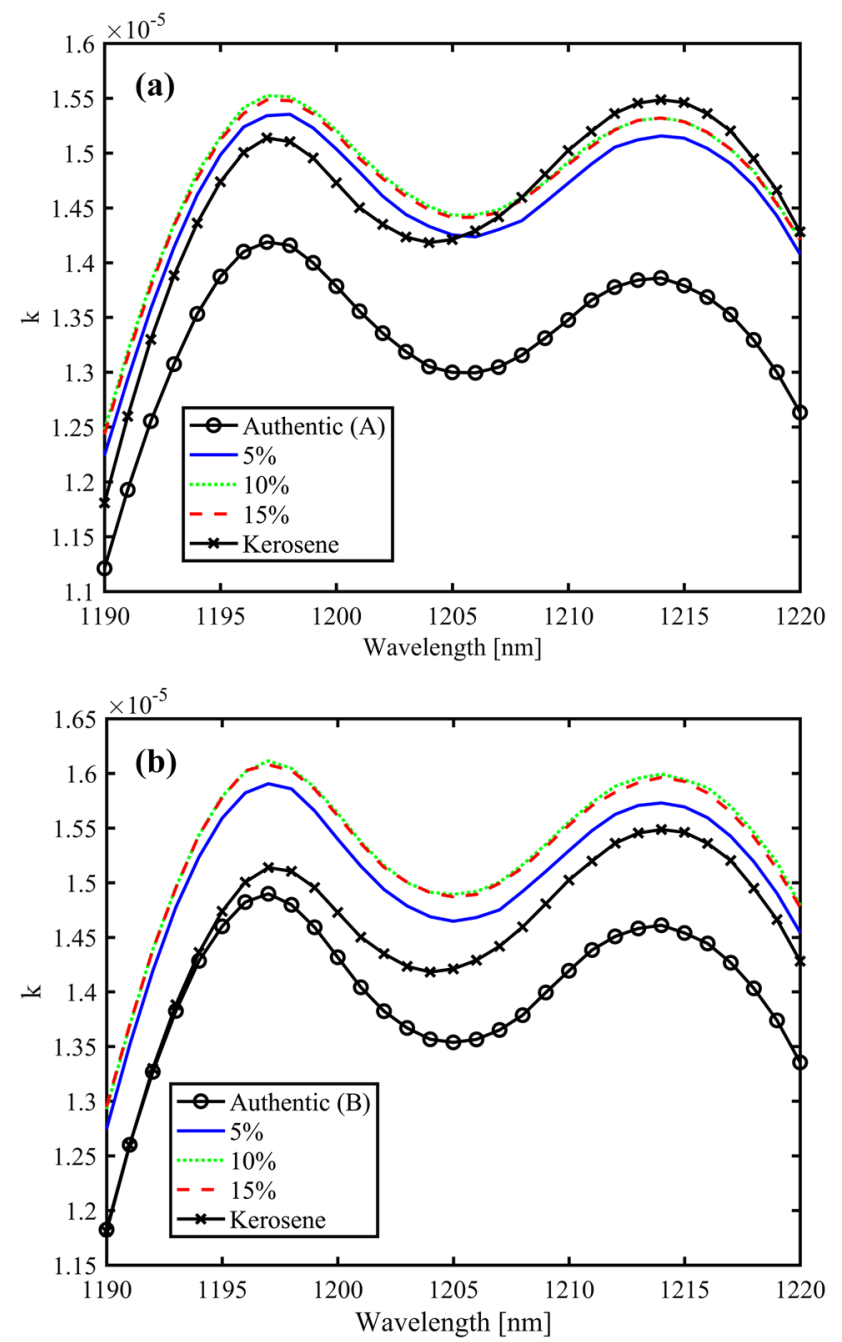

Fig. 2 Magnifications of extinction coefficient $k(\lambda)$ curves for kerosene and authentic and adulterated samples in the vicinity of 1190 to $1220 \mathrm{~nm}$. a Sample A, b sample B kerosene cannot be judged from the relative heights in this spectral region, because the left shoulder is always higher, thus emphasizing the presence of diesel oil. It is also clear from Fig. 2a, b that for $10 \%$, and $15 \%$-adulterated samples, the extinction coefficient curves overlap, and it is difficult to find differences because of the relative heights of the two nearby bands of diesel oil and kerosene. Hence, there is no clear rule that enables one to use the extinction coefficient to predict the adulteration percentage.

Figure $3 \mathrm{a}, \mathrm{b}$ shows magnifications of the extinction coefficient curves for kerosene, authentic sample A, and authentic sample B with their corresponding adulteration percentages in the spectral range 1390-1427 nm. In contrast to the observation made for the fingerprints between 1190 and $1220 \mathrm{~nm}$, where the difference in the relative height exists between the nearby bands for diesel oil and kerosene, such difference
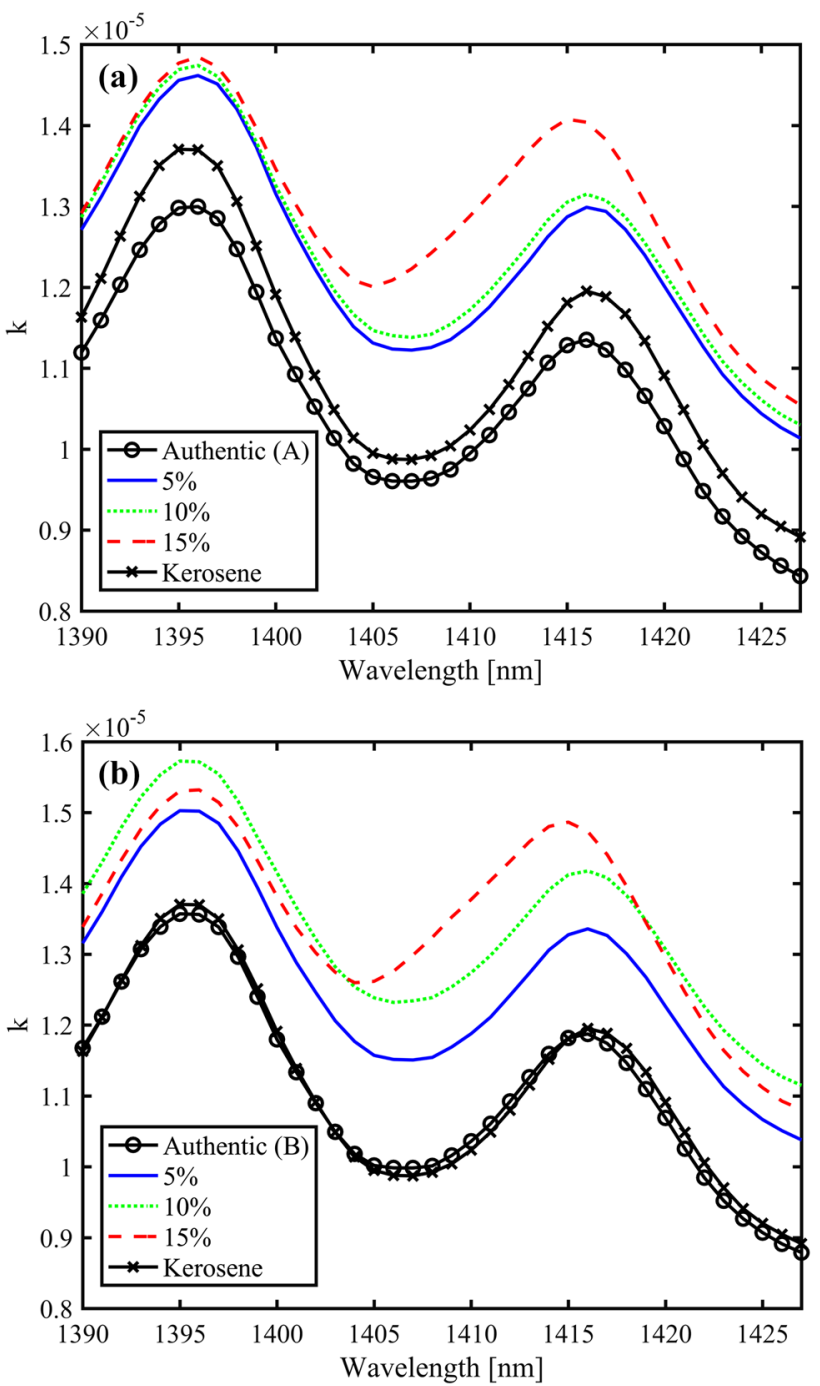

Fig. 3 Magnifications of extinction coefficient $k(\lambda)$ curves for authentic and adulterated samples in the vicinity of 1390-1427 nm. a Sample A, b sample B 
in the relative height is not present for the two bands in the vicinity of the strong absorption ranging from 1395 to $1415 \mathrm{~nm}$. In this spectral range, the maximum extinction coefficient curves at approximately $1395 \mathrm{~nm}$ and $1415 \mathrm{~nm}$ increase with increasing adulteration percentage. This is clear for both adulterated samples A and B in this spectral region. Furthermore, the curves of the extinction coefficient of authentic sample B and kerosene in Fig. $3 \mathrm{~b}$ almost completely overlap, but the curves for the adulterated samples are much higher. For authentic sample A and kerosene, in Fig. 3a, both curves are well resolved, but after mixing kerosene and diesel oil, the extinction coefficient curves for the adulterated samples are higher. The maximum extinction coefficient increases with increasing adulteration percentage, which is clearest in the vicinity of $1415 \mathrm{~nm}$. Hence, from the extinction coefficient curves in Fig. 3a, b, we can see an excess value of $k$, namely, the interaction of the molecules of the two fuels. From Figs. 2 and 3, it is evident that $1390-1420 \mathrm{~nm}$ is optimal for screening the adulterated fuels.

According to refractive index readings in Table 1 , if we have a relatively highly adulterated sample $15 \%$ in this study, we can dilute it first to $10 \%$ and then to $5 \%$, with authentic diesel to obtain more information about it. Note that the operating wavelength of the Abbe refractometer is $589 \mathrm{~nm}$, whereas we monitor the extinction coefficient curves, such as those presented in Fig. 3a, b, at much higher wavelengths. Then, the extinction coefficient curves complement the prediction based on the readings of the Abbe refractometer, and we obtain the adulterated samples in the correct order of adulteration from the spectrophotometer readings at 1390-1420 nm. Although the Abbe refractometer reading for sample B with $15 \%$ adulteration, which is 1.44731 , could lead to it being accepted as authentic diesel oil, because it is close to the refractive index of authentic diesel B, namely, 1.44775 , the extinction coefficient data clearly show that it is an adulterated sample. We can thus use both Abbe refractometer data and extinction coefficient data (at $1400 \mathrm{~nm}$ ) to screen adulterated diesel oils. To determine the excess extinction coefficient using the expression $k(\lambda)-k^{\text {ideal }}(\lambda)$, the extinction coefficient is applied. However, to clearly distinguish and discriminate between samples with different adulteration percentages, we employ a rigorous theory, given in [15], associated with excess permittivity. Therefore, in this work, in addition to the excess extinction coefficient, we introduce one more measure, which is based on the calculation of the imaginary excess permittivity of diesel oil and kerosene mixtures. As already concluded from the magnified extinction coefficient curves, the optimum spectral range for examining imaginary excess permittivity is in the vicinity of $1395-1415 \mathrm{~nm}$. The imaginary excess permittivity was calculated using Eqs. (4), (6), and (7) within this spectral range.

Figure $4 \mathrm{a}, \mathrm{b}$ shows estimates for the refractive index of the adulterated fuel samples calculated using Eq. (7) and
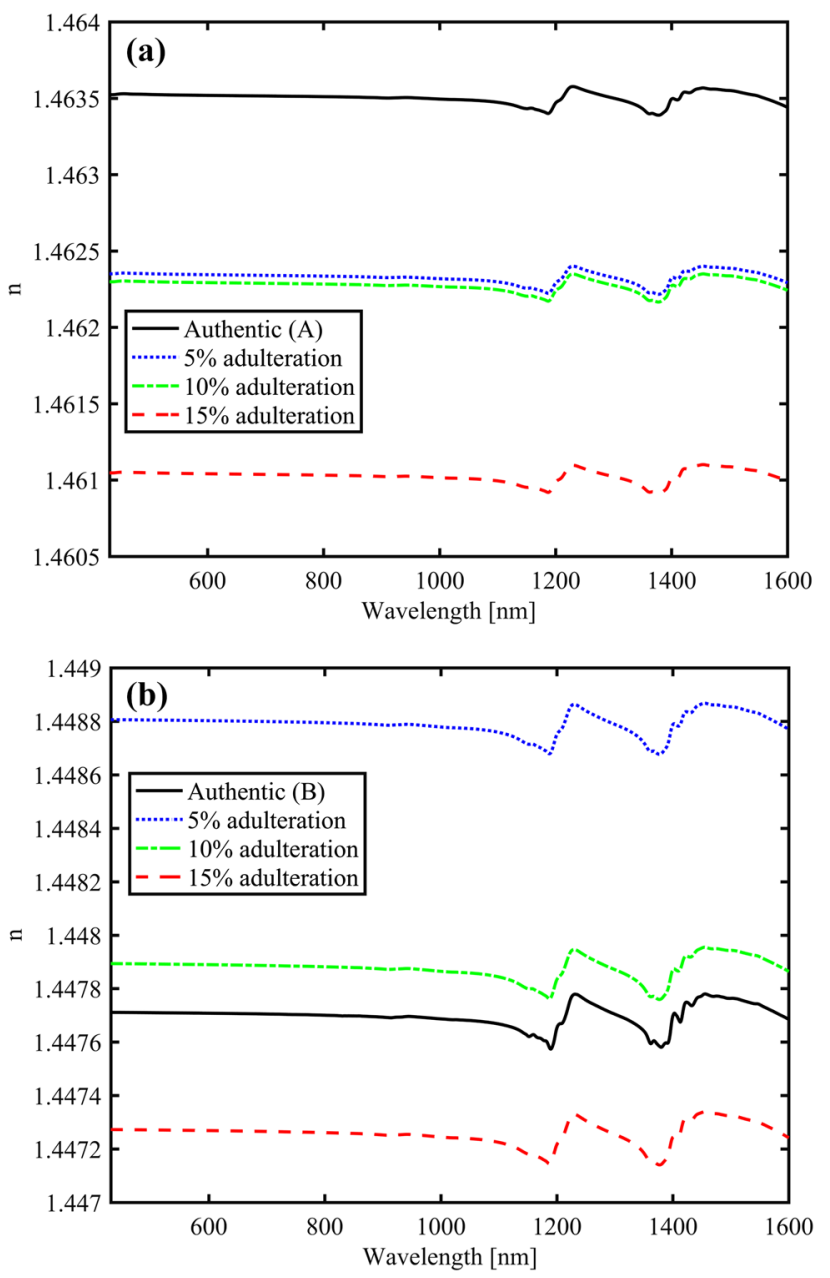

Fig. 4 Wavelength dependence of refractive index $n(\lambda)$ for a adulterated sample A, b adulterated sample B

by finite-range integration. These data were used to calculate the imaginary excess permittivity. From Fig. 4a, b, it is clear that the greatest change in the refractive index is in the region of anomalous dispersion in the NIR absorption bands. The SSKK relation gives stronger convergence of the integral than the conventional Kramers-Kronig relation; hence, the accuracy of data inversion is high even for finite-range spectral data. Our aim is to develop an optical sensor that only uses measured finite spectral data in the analysis method to screen adulterated diesel oils. Successful data inversion using the SSKK relation in the case of a narrow spectral range and omission of the nearby spectral fingerprint was demonstrated in [22]. Recently, the validity of the method using the finite-range SSKK relation has been demonstrated even for saturated absorption bands, by simulations of absorption with a Lorentzian in [23].

Next, Fig. 5a shows the wavelength dependence of the imaginary excess permittivity of adulterated sample A in the spectral range of $1000-1600 \mathrm{~nm}$, where the curves were 

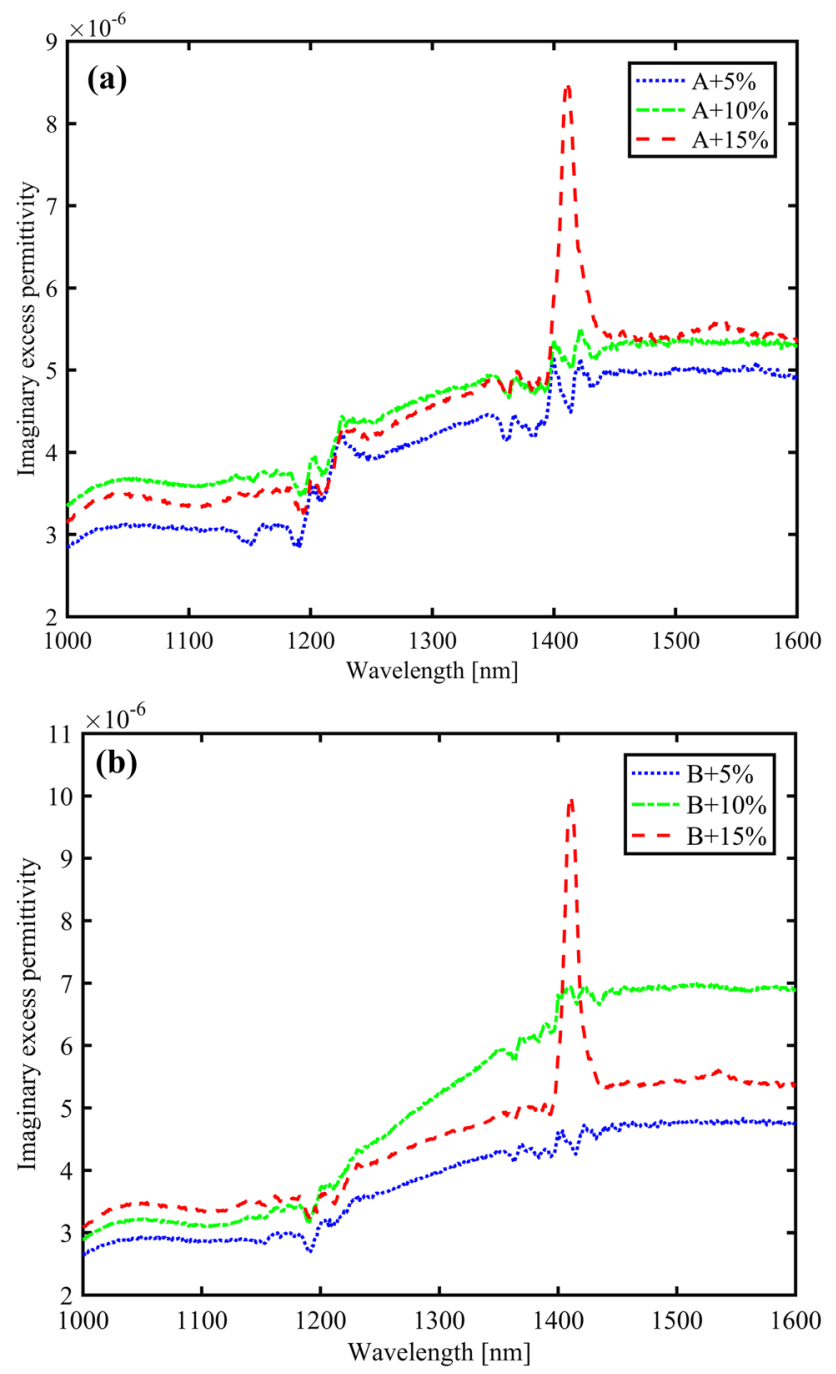

Fig. 5 Wavelength dependence of imaginary excess permittivity Im $\varepsilon^{\mathrm{E}}(\lambda)$ for samples adulterated by different volume fractions of kerosene. a Sample A, b sample B

obtained as the difference between the imaginary permittivity of the adulterated samples and that of the ideal binary mixtures. It is clear from Fig. 5a that 5\% adulteration has the lowest imaginary excess permittivity, followed by $10 \%$ adulteration. Furthermore, the sample adulterated with $15 \%$ kerosene has a much higher imaginary excess permittivity than the samples with 5\% and $10 \%$ adulteration. This demonstrates that using the imaginary excess permittivity, it is possible to separate and discriminate sample A adulterated with different volume fractions of kerosene. Furthermore, Fig. $5 \mathrm{~b}$ shows the wavelength dependence of the imaginary excess permittivity of adulterated sample B in the spectral range of $1000-1600 \mathrm{~nm}$. In this case, we observe a similar behavior for 5\% and 10\% adulteration to that in Fig. 5a: one can distinguish two nearby peaks of excess imaginary permittivity that reflect the two absorption bands. However, a single strong peak is observed for $15 \%$ adulteration in Fig. 5a, b, which implies that this is not merely a coincidence, because it is present for both diesel oils. The baseline of $10 \%$ in Fig. $5 \mathrm{~b}$ is strikingly high compared with that in Fig. 5a. However, the 15\% adulterated sample in Fig. 5a, b can be judged as an adulterated diesel oil owing to its higher peak. The imaginary excess permittivity can be used in decision making to screen diesel oil adulteration by kerosene.

As mentioned already, sample B has a refractive index very close to that of kerosene, and this further complicates the detection of adulteration using only Abbe refractometer readings, because the similar values increase the uncertainty. Hence, from the information of the refractive index measured by the Abbe refractometer, the extinction coefficient obtained from spectrophotometer measurements, as well as the imaginary excess permittivity obtained by a combination of these data through SSKK analysis, we can not only decide with certainty the presence of adulterated diesel oil, but also identify and discriminate low from high levels of adulteration.

In Fig. 5a, b, a strong peak is observed for $15 \%$ adulteration, whereas for $5 \%$ and $10 \%$ adulteration, a strong peak is not present. We suggest that this type of curve provides evidence for the abnormal dependence of the imaginary excess permittivity on the kerosene fill fraction and hence supports the observation that the $\mathrm{L}-\mathrm{L}$ formula is invalid for adulterated diesel samples, especially when the amount of kerosene is relatively large [7]. The $\mathrm{L}-\mathrm{L}$ formula for permittivity, indicating a linear dependence on the fill fraction of a foreign liquid, is valid in the absence of chemical activity of the liquids involved in the binary mixture. On the other hand, the interaction between different hydrocarbons, as well as other molecules that have been added to the diesel oil in the refinery, may cause various interactions with the molecules present. Therefore, the abnormal behaviors of the extinction coefficient and imaginary permittivity are due to the chemical activity of the mixed liquids. This, in turn, may explain the abnormal dependence of the imaginary excess permittivity on the kerosene fill fraction.

For practical field measurements, the most important task is to detect adulterated diesel oil. Hence, accurate knowledge of the kerosene fill fraction is a secondary issue. Since the origin of the crude oil, its refining to obtain diesel oil, and the additives used may result in chemical activity with kerosene, it is difficult to find a general rule for predicting the kerosene fill fraction, and the present study gives only a partial solution. Both experimental and theoretical investigations are needed to find a rule to predict the kerosene percentage from the imaginary excess permittivity.

In our recent article, we have shown that conventional kerosene and diesel oils from different origins show optical properties such as excess refractive index and excess permittivity [7]. However, excess permittivity, being a more 
fundamental quantity, is usually preferable. Furthermore, the wavelength dependence of imaginary excess permittivity can be used to estimate, for instance, the contact angle of binary mixtures, which is important in the development of a handheld sensor [6] for screening adulterated diesel oil.

In the case that markers are used, such as polar markers or impurities, for the identification of adulterated diesel oils, as suggested in [24], these markers contribute to the permittivity of diesel oil and kerosene and thus to the excess permittivity. Hence, the excess permittivity is a useful and relatively accessible measure for screening adulterated diesel oil containing fingerprints in the presence of polar markers.

The volume of liquid needed for the measurement of the liquid fuel is small: only a drop is required for measurement with an Abbe refractometer or the volume of a thin spectroscopic cuvette is required. In principle, it is possible to combine a refractometer and a spectrophotometer in a single measurement unit, as described in [25], and utilize the concept of the imaginary excess permittivity for the data. In such a case, only one liquid measurement compartment is required.

\section{Conclusion}

In this work, we have studied the wavelength dependence of the imaginary optical properties of diesel oils adulterated with different volume fractions of kerosene in the NIR spectral range using general dispersion analysis based on Kramers-Kronig analysis. We have demonstrated that using both the extinction coefficient and imaginary excess permittivity, it is possible to obtain extra information about diesel oils adulterated with different volume fractions of kerosene, particularly in situations, where the refractive indices of diesel oil and kerosene are very close for a fixed measurement wavelength of the probe light. In addition, when the volume of kerosene was $15 \%$ which is below the typical adulteration level of $20 \%$, a strong peak of imaginary excess permittivity was observed, which was very different from the cases of $5 \%$ and $10 \%$ adulteration. Hence, the extinction coefficient and excess imaginary permittivity complement each other in the screening of diesel oil for adulteration. Moreover, the imaginary permittivity can also be exploited for the development of practical sensors for the screening of diesel oil adulteration.

Acknowledgements Open access funding provided by University of Eastern Finland (UEF) including Kuopio University Hospital. The authors would like to thank Dr. Prince Bawuah for his support with the SSKK calculations and Dr. Martti Makinen for his assistance during the measurements.

\section{Compliance with ethical standards}

Conflict of interest The authors declare no conflict of interest.

Open Access This article is distributed under the terms of the Creative Commons Attribution 4.0 International License (http://creativeco mmons.org/licenses/by/4.0/), which permits unrestricted use, distribution, and reproduction in any medium, provided you give appropriate credit to the original author(s) and the source, provide a link to the Creative Commons license, and indicate if changes were made.

\section{References}

1. Mishra, V., Jain, S.C., Singh, N., Poddar, G., Kapur, P.: Fuel adulteration detection using long period fiber grating sensor technology. IJPAP 46, 106-110 (2008)

2. Pathak, A.K., Gangwar, R.K., Priyadarshini, P., Singh, V.K.: A robust optical fiber sensor for the detection of petrol adulteration. Optik 149, 43-48 (2017)

3. Taksande, A., Hariharan, C.: Synchronous fluorescence method to check adulteration of petrol and diesel by kerosene. Spectrosc. Lett. 39, 345-356 (2006)

4. Bassbasi, M., Hafid, A., Platikanov, S., Tauler, R., Oussama, A.: Study of motor oil adulteration by infrared spectroscopy and chemometrics methods. Fuel 104, 798-804 (2013)

5. Paiva, E.M., Rohwedder, J.J.R., Pasquini, C., Pimentel, M.F., Pereira, C.F.: Quantification of biodiesel and adulteration with vegetable oils in diesel/biodiesel blends using portable near-infrared spectrometer. Fuel 160, 57-63 (2015)

6. Kanyathare, B., Kuivalainen, K., Räty, J., Silfsten, P., Bawuah, P., Peiponen, K.-E.: A prototype of an optical sensor for the identification of diesel oil adulterated by kerosene J. Eur. Opt. Soc. Rapid Publ. 14, 1-6 (2018)

7. Kanyathare, B., Peiponen, K.-E.: Hand-held refractometer based measurement and excess permittivity analysis method for detection of diesel oils adulterated by kerosene in field conditions. Sensors 18, 1551 (2018)

8. Betancourt, S.S., Bracey, J., Gustavson, G., Mathews, S.G., Mullins, O.A.: Chain of custody for samples of live crude oil using visible-near-infrared spectroscopy. Appl. Spectrosc. 60, 14821487 (2006)

9. Kanyathare, B., Peiponen, K.-E.: Wavelength-dependent excess permittivity as indicator of kerosene in diesel oil. Appl. Opt. 57, 2997-3002 (2018)

10. Reis, J.C., Iglesias, T.P., Douhéret, G., Davis, M.J.: The permittivity of thermodynamically ideal liquid mixtures and the excess relative permittivity of binary dielectrics. Phys. Chem. Chem. Phys. 11, 3977-3986 (2009)

11. Hadded, N., Bouanz, M.: Excess permittivity and excess conductivity of binary fluid mixture isobutyric acid-water along the coexistence curve. Phys. Chem. Liq. 47, 160-171 (2009)

12. Baranović, G.: Refractive index mixing rules and excess infrared spectra of binary mixtures. Appl. Spectrosc. 71, 1039-1049 (2017)

13. McGregor, J., Li, R., Zeitler, J.A., Agostino, C.D., Collins, J.H.P., Mantle, M.D., Manyar, H., Holbrey, J.D., Falkowska, M., Youngs, T.G.A., Hardacre, C., Stiff, E.H., Gladden, L.F.: Structure and dynamics of aqueous 2-proponol: a THz-TDS, NMR and neutron diffraction study. Phys. Chem. Chem. Phys. 17, 30481-30491 (2015)

14. Mou, S., Rubano, A., Paparo, D.: Complex permittivity of ionic liquid mixtures investigated by terahertz time-domain spectroscopy. J. Phys. Chem. B 121, 7351-7358 (2017) 
15. Iglesias, T.P., Reis, J.C.R.: On the definition of the excess permittivity of a fluid mixture III dependence on frequency. J. Chem. Thermodyn. 96, 208-209 (2016)

16. Lucarini, V., Saarinen, J.J., Peiponen, K.E., Vartiainen, E.M.: Kramers-Kronig Relations in Optical Materials Research. Springer, Berlin (2005)

17. Szymkowice, P.G., Benoges, J.: Development of diesel surrogate fuel library. Fuel 222, 21-34 (2018)

18. Wiener, O.: Die Theorie des Mischkörpes für das Feld der Stationären Strömung. Abh. Math. Phys. Kl. König Ges. 32, 509-604 (1912)

19. Palmer, K.F., Williams, M.Z., Budde, B.A.: Multiply subtractive Kramers-Kronig relations. Appl. Opt. 37, 2660-2673 (1998)

20. Dombrovsky, L.A., Sazhin, S.S., Mikhalovsky, S.U., Wood, R., Heikel, M.R.: Spectral properties of diesel fuel droplets. Fuel 82, 15-22 (2003)

21. Chung, H., Ku, M.-S., Lee, J.S.: Comparison of near-infrared and mid-infrared spectroscopy for the determination of distillation property of kerosene. Vibr. Spectrosc. 20, 155-163 (1999)
22. Lucarini, V., Bassani, F., Peiponen, K.-E., Saarinen, J.J.: Dispersion theory and sum rules in linear and nonlinear optics. La Rivista del Nuovo Cimento. 26, 1120 (2003)

23. Bernier, M., Garet, F., Coutaz, J.-L., Minamide, H., Sato, A.: Accurate characterization of resonant samples in terahertz regime through a technique combining time-domain spectroscopy and Kramers-Kronig analysis. IEEE Trans. Terahertz Sci. Technol. 6, 442-450 (2016)

24. Haddad, R., Regiani, T.C., Klitzke, C.F., Sanvido, G.B., Corilo, Y.E., Augusti, D.V., Pasa, V.M.D., Pereira, R.C.C., Romao, W., Vaz, B.G., Augusti, R., Eberdin, M.N.: Gasoline, kerosene, and diesel fingerprinting via polar markers. Energy Fuels 26, 35423547 (2012)

25. Niskanen, I., Räty, J., Peiponen, K.E.: A multifunction spectrophotometer for measurement of optical properties of transparent and turbid liquids. Meas. Sci. Technol. 17, 87-91 (2007) 